\title{
Contralateral pneumothorax after cardiac pacemaker implantation
}

\author{
Małgorzata Hardzina ${ }^{1}$, Andrzej Ząbek ${ }^{1}$, Krzysztof Boczar ${ }^{1}$, Paweł Matusik ${ }^{1}$, Barbara Małecka ${ }^{1,2}$, Jacek Lelakowski ${ }^{1,2}$ \\ ${ }^{1}$ Department of Electrocardiology, The John Paul II Hospital, Krakow, Poland \\ ${ }^{2}$ Institute of Cardiology, Jagiellonian University, Medical College, Krakow, Poland
}

Postep Kardiol Inter 2015; 11, 4 (42): 347-348

DOI: $10.5114 /$ pwki.2015.55611

We report a case of a 73-year-old man, who was scheduled for elective dual chamber pacemaker implantation because of paroxysmal second-degree atrioventricular block. The pacemaker pocket was located in the left infraclavicular fossa. An active fixation ventricular lead was implanted into the right ventricular outflow tract (RVOT) through the left cephalic vein venesection, while another active fixation (Medtronic CapSure 5076MRI) atrial lead was located within the right atrial appendage using left subclavian vein puncture.

During the procedure, due to anatomical inconveniences, several attempts at subclavian vein puncture were performed. Difficulties were also encountered during lead introduction through the superior vena cava. At the end of the procedure spontaneous dislocation of the atrial lead occurred. The young doctor repositioned this lead into the free wall of the right atrium (RA). Optimal sensing, pacing, and impedance parameters for both leads were obtained.

The day after operation, the patient reported unspecific pain in the upper abdominal region and later strong, intermittent, stabbing pain in the lower chest. These symptoms intensified during inspiration and while lying on left side. The patient negated dyspnoea. On auscultation, normal vesicular breath sounds were heard. Neither pericardial fluid nor signs of RV perforation were found in transthoracic echocardiography (TTE). In the telemetric pacemaker assessment an increased pacing threshold of atrial lead was noticed (from $<0.5 \mathrm{~V} / 0.4 \mathrm{~ms}$ during pacemaker implantation to $4.5 \mathrm{~V} / 1 \mathrm{~ms}$ in unipolar pacing and $3 \mathrm{~V} / 1 \mathrm{~ms}$ in bipolar pacing); no changes in sensing or impedance parameters were found. However, due to the lack of exact assessment of the atrial lead tip positioning, it was impossible to definitively exclude RA perforation (Figure $1 \mathrm{~A}$ ). The device was reprogrammed to bipolar stimulation, leading to a decrease in reported complaints.

On the next day a lack of atrial lead capture by threshold $>7.5$ V/1.5 ms was recorded. Fluoroscopy revealed dislocation of the atrial lead, which was extracted subsequently. New lead was implanted through subclavian vein puncture into the right atrial appendage, obtaining good parameters (Figures 1 B, C). Several hours after reoperation the patient reported resting dyspnoea. Chest radiograph revealed large right-sided pneumothorax (Figure 1 D), which was cured by suction drainage.

There are two probable causes of contralateral pneumothorax in the reported case. The first is pleura injury during Seldinger set introduction. Taking into account potential small mediastinal bleeding size, the venous system (especially the extrapericardial part of the superior vena cava) puncture could be done by guidewire (part of the Seldinger set). The second is right atrium, pericardium, and pleura perforation by a primary implanted atrial lead that dislocated or was extracted with subsequent pneumothorax. Because of the lack of fluid excess signs in the pericardial sac (during both physical and radiological examination), the first hypothesis seems more likely. However, contralateral pleural puncture during subclavian vein cannulation cannot be excluded.

Treatment by chest tube placement and/or pacing lead reposition or extraction in case of heart perforation seem to provide satisfactory results in most patients with contralateral pneumothorax after pacemaker implantation [1-4]. In some cases a new atrial lead (also epicardial) was implanted and/or atrial or pericardial repair was performed [1, 3].

\section{Corresponding author:}

Paweł Matusik MD, PhD, Department of Electrocardiology, The John Paul II Hospital, 80 Prądnicka St, 31-202 Krakow, Poland, phone: +48 1261422 77, fax: +48 1263323 99, e-mail: pawel.matusik@wp.eu

Received: 19.03 .2014 , accepted: 14.07.2015. 

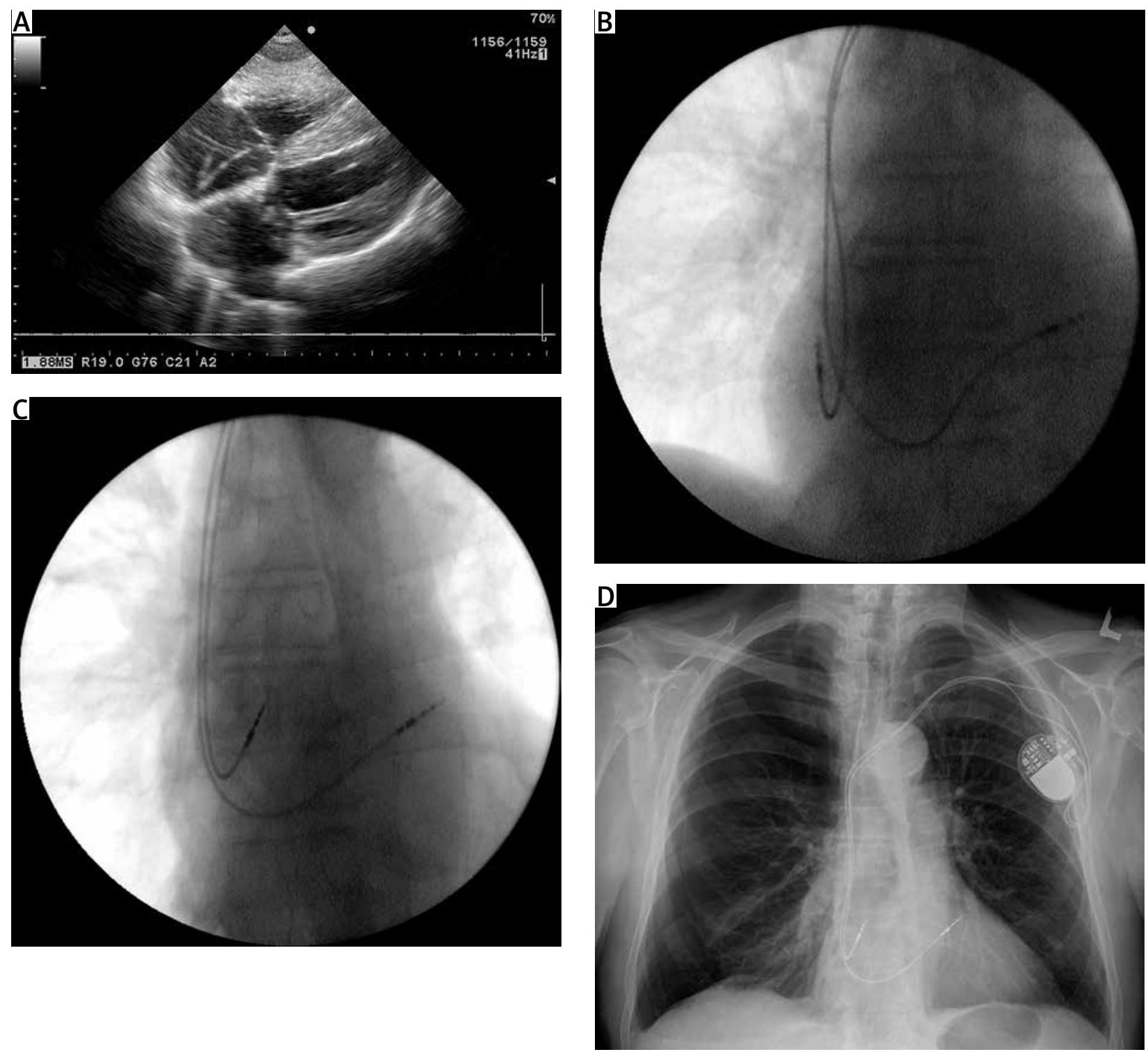

Figure 1. A - Echocardiographic study showing parts of the atrial and ventricular lead and no signs of pericardial fluid excess. B - Fluoroscopy after first implantation. C - Fluoroscopy after spontaneous dislocation of atrial lead. D - Right-sided pneumothorax after pacemaker implantation

\section{Conflict of interest}

The authors declare no conflict of interest.

\section{References}

1. Dilling-Boer D, Ector H, Willems R, Heidbuchel H. Pericardial effusion and right-sided pneumothorax resulting from an atrial active-fixation lead. Europace 2003; 5: 419-23.

2. Van Herendael H, Willems R. Contralateral pneumothorax after endocardial dual-chamber pacemaker implantation resulting from atrial lead perforation. Acta Cardiol 2009; 64: 271-3.

3. Srivathsan K, Byrne RA, Appleton CP, Scott LR. Pneumopericardium and pneumothorax contralateral to venous access site after permanent pacemaker implantation. Europace 2003; 5: 361-3.

4. Pettemerides V, Jenkins N. Contralateral pneumothorax following repositioning of an atrial lead. Europace 2012; 14: 606. 